\title{
Single allele mutations at the heart of congenital disease
}

\author{
Nadia Rosenthal ${ }^{1}$ and Richard P. Harvey ${ }^{2,3}$ \\ ${ }^{1}$ Cardiovascular Research Center, Massachusetts General Hospital-East, Charlestown, Massachusetts 02129, USA \\ 2Victor Chang Cardiac Research Institute, Darlinghurst, Sydney 2010, Australia \\ ${ }^{3}$ Faculties of Medicine and Life Sciences, University of New South Wales, Kensington, Sydney 2052, Australia
}

Address correspondence to: Nadia Rosenthal, Cardiovascular Research Center, Massachusetts General Hospital, 149 13th Street, Charlestown, Massachusetts 02129, USA. Phone: (617) 724-9560; Fax: (617) 724-9561; E-mail: rosenthal@helix.mgh.harvard.edu.

A prevailing dream among today's developmental biologists is that the genes they discover controlling basic embryonic processes in animal models will eventually surface in mutant form as the cause of human genetic disorders. This hope has now been realized for the NKX2.5 gene, which encodes a member of the homeobox transcription factor family that is expressed in cardiac muscle during embryonic, fetal, and adult life (1). In the 7 years since it was first cloned from the mouse, $N k \times 2.5$ has emerged as a key regulator of cardiac development in numerous vertebrate model systems, even those with the most primitive hearts $(2,3)$. In genetically engineered mice lacking both copies of the $N k \times 2.5$ gene, heart development is arrested, and the heart remains a primitive linear tube, which fails to loop or to develop definitive chambers (1).

The early lethality caused by mutation of Nkx2.5 in mice is not surprising, given that its homologue tinman is absolutely essential for formation of the muscular heartlike structure in the fruit fly Drosopbila (1). The number of downstream cardiac regulatory genes shown to be dependent upon Nkx2.5 has placed this gene near the top of a genetic hierarchy responsible for heart development in vertebrates.

The disruption of a gene in laboratory mice involves first producing heterozygous offspring that carry a single null allele. The apparently normal cardiac development in mice heterozygous for an Nkx2.5 targeted mutation initially led researchers to believe that the effects of its disruption were recessive, but this view is now challenged by two recent clinical studies (one in this issue of the JCI) reporting that multiple congenital heart defects can be ascribed to inherited mutations in one allele of the human NKX2.5 gene $(4,5)$.
Diverse cardiac defects in families with NKX2.5 mutations. Several NKX2.5 mutations have been discovered in families affected with cardiac defects. Although the functional impact of some of these mutations is uncertain, others, affecting amino acids within the DNA-binding homeodomain, would certainly be disruptive to protein function. Unlike well-characterized dominant conditions such as Holt-Oram syndrome (6), in which cardiac and forelimb abnormalities are prominent features, specific NKX2.5 alleles do not appear to cosegregate with particular clinical phenotypes. A wide variety of cardiac malformations appear in these families, predominantly atrial septal defect and atrioventricular conduction block, but also ventricular septal defects, tricuspid valve abnormalities, double-outlet right ventricle, and tetralogy of Fallot, a common cause of la maladie bleue (blue babies). Ebstein's malformation, a displacement of the hinge-points of tricuspid valve leaflets, is now for the first time associated with a genetic locus (4).

Together, these studies represent a significant breakthrough in our understanding of human heart defects. Cardiac abnormalities have been ascribed more often to complex interactions between genes and environment than to mendelian inheritance of single gene mutations. This perspective may now need to be reevaluated. Single-allele mutations underlying congenital abnormalities may be masked by factors such as the less-than-perfect cosegregation of mutation and malformation (partial penetrance), variability in severity and nature of the abnormality (partial expressivity), and asymptomatic individuals seen in affected families (7).

In the wake of human studies, developmental biologists were quick to reexamine mouse Nkx2.5 heterozy- gotes, looking for clues to the phenotypic variability seen in families with NKX2.5 mutations. In one mouse strain, preliminary studies have revealed atrial septal defects in $20 \%$ of Nkx2.5 heterozygotes, along with conduction abnormalities (S. Izumo, personal communication). In the original knockout line, only milder atrial septal defects were found (R.P. Harvey, unpublished data). Whether these differences are due to genetic background or to the nature of the targeted mutation is not yet clear. However, it has become evident that background genetic differences be-tween individuals can profoundly modify the effect of a particular mutation. In Nkx2.5 knockout experiments, such modifier genes that would be identical between individual laboratory mice kept on genetically homogeneous inbred backgrounds might well vary between mice with different inbred backgrounds. The genetic homogeneity of laboratory mice contrasts with the genetic heterogeneity of human populations, but represents a powerful jumping-off point for tracking down the identity of genetic modifiers that may alter the nature or severity of defects in humans.

Retbinking haploinsufficiency. Although modifier genes no doubt underlie some of the variability in $N K X 2.5$-related defects, the mouse studies hint at other causes. Abnormalities resulting from single null mutations are attributed to haploinsufficiency, a term that suggests that 2 -fold reduction of expression underlies the observed defect. For genes such as NKX2.5 that encode transcription factors, haploinsufficiency is usually defined as a below-threshold level of protein produced from the single normal allele, leading to pathologically low levels of expression of downstream target genes. The molecular conse- 
quences of haploinsufficiency may need to be reinterpreted in light of accumulating evidence that gene expression is controlled in a probabilistic manner. Thus, transcriptional enhancers, which bind transcription factors, are thought to increase only the probability of gene activation within a given cell, rather than the level of expression (8). The implications of this slight change in emphasis are profound: a low level of NKX2.5 protein expressed in heterozygotes may mean that some cells, on a probabilistic basis, will not activate target genes at all, or will activate them only sporadically. Developmental events that operate in a small number of cells, or that occur during a narrow temporal window may be especially prone to the effects of disrupting single alleles of regulatory genes. To complicate matters, an increasing number of transcription-factor genes are being found that show random monoallelic expression, i.e., only one allele is ever active in any one cell (9). In individuals who are heterozygous for a mutation, some cells may express the functional allele, but others express only the mutant allele, and therefore no functional protein at all. If these mechanisms are operative in NKX2.5 heterozygotes, cells that activate NKX2.5 target genes during heart development may be randomly determined, and may therefore vary enormously in number and spatial distribution between affected individuals. In this way, probabilistic mechanisms of gene expression could have profound implications for the morphological outcome of single allele mutations.

The range of defects in affected families also suggests that the NKX2.5 transcription factor participates in a number of distinct morphogenetic processes in heart development that were previously obscured in homozygous mice due to early lethality. Although this notion is intriguing, the observed defects could also reflect milder manifestations of the arrest of looping seen in mouse Nkx2.5 homozygotes, with individual outcomes determined by modifier genes and probabilistic mechanisms. Looping sets the stage for many downstream remodeling events in heart development, and failure to complete looping has been proposed to explain the impressive variety of cardiac defects seen in trisomy 16 mice, which represent a partial model of Down's syndrome (10). For the moment, therefore, we should not assume that all abnormal structures arising from NKX2.5 mutation reflect a direct role for the gene in their formation.

What are the clinical implications of these studies? Genetic screening for NKX2.5 mutations within families with congenital abnormalities would be of clear prognostic value in prenatal counseling. Whether familial NKX2.5 mutations turn out to be more frequent than expected in the general population, and whether NKX2.5 mutations are significant genetic factors in idiopathic cases will require further analysis. Nevertheless, the issues raised by these studies are likely to generate new principles relevant to a wide spectrum of dominant human genetic diseases.

\section{Acknowledgments}

The authors wish to thank Seigo Izumo for sharing unpublished results.

1. Harvey, R.P. 1996. NK-2 homeobox genes and heart development. Dev. Biol. 178:203-216.

2. Fu, Y., Yan, W., Mohun, T.J., and Evans, S.M. 1998. Vertebrate tinman homologues $X N k \times 2-3$ and $X N k \times 2-5$ are required for heart formation in a functionally redundant manner. Development. 125:4439-4449.

3. Grow, M.W., and Krieg, P.A. 1998. Tinman function is essential for vertebrate heart development: elimination of cardiac differentiation by dominant inhibitory mutants of the tinman-related genes, XNkx2-3 and XNkx2-5. Dev. Biol. 204:187-196.

4. Benson, D.W., et al. 1999. Mutations in the cardiac transcription factor $N K X 2.5$ affect diverse cardiac developmental pathways. J. Clin. Invest. 104:1567-1573

5. Schott, J.-J., et al. 1998. Congential heart disease caused by mutations in the transcription factor NKX2-5. Science. 281:108-111.

6. Basson, C.T., et al. 1999. Different TBX5 interactions in heart and limb defined by Holt-Oram syndrome mutations. Proc. Natl. Acad. Sci. USA. 96:2919-2924.

7. Benson, D.W., et al. 1998. Reduced penetrance, variable expressivity, and genetic heterogeneity of familial atrial septal defects. Circulation. 97:2043-2048.

8. Fiering, S., Whitelaw, E., and Martin, D.I.K. 1999. To be or not to be active: the stochastic nature of enhancer action. Bioessays. In press.

9. Nutt, S.L., et al. 1999. Independent regulation of the two Pax5 alleles during B-cell development. Nat. Genet. 21:390-395.

10. Mjaatvedt, C.H., et al. 1998. Mechanisms of segmentation, septation, and remodelling of the tubular heart: endocardial cushion fate and cardiac looping. In Heart development. R.P. Harvey and N. Rosenthal, editors. Academic Press. San Diego, CA. $159-177$. 